\title{
Evaluating the effect of changes in bone geometry on the trans-femoral socket-residual limb interface using finite element analysis
}

\author{
Alex van Heesewijk ${ }^{1}$, Andy Crocombe ${ }^{1}$, Serge Cirovic ${ }^{1}$, Mathew Taylor ${ }^{1}$, and Wei \\ $\mathrm{Xu}^{1}$ \\ ${ }^{1}$ University of Surrey, Guildford, Surrey, GU2 7XH, UK \\ a.vanheesewijk@surrey.ac.uk
}

\begin{abstract}
A prosthetic socket used by a lower limb amputee should accommodate the patient's geometry and biomechanical needs. The creation of a geometrically accurate subject-specific finite element model can be used to provide a better understanding of the load transfer between socket and limb. There has been a limited number of finite element studies of trans-femoral sockets with all current models only including the femur and ignoring the pelvis. This study looked to evaluate the effect that including the pelvic bone as well as the femur in a finite element model has on the contact interface between the prosthetic socket and residual limb. This was done by creating a finite element model from a computerised tomography scan of a trans-femoral amputee. This model included threedimensional geometry, nonlinear material properties and frictional contact between the residual limb and prosthetic socket. It was found that without the pelvic bone the contact pressures peaked at the distal end region of the residual limb (peak of $95 \mathrm{kPa}$ ). However by including the pelvic bone the contact pressures were instead concentrated at the ischial loading region (peak of $364 \mathrm{kPa}$ ). The shear stresses experienced on the socket-residual limb interface were also simulated. The results obtained in this study can be used to provide more of an understanding of the loading on the residual limb for the design and creation of future trans-femoral sockets.
\end{abstract}

Keywords: Finite element analysis, Trans-femoral, Prosthetic socket

\section{Introduction}

The amputation of a lower limb is a severely traumatic experience. The prosthetic solution to this aims to restore the self-esteem of the patient as well as to restore the best ambulation achievable for the patient. The prosthetic socket introduces an interface between the residual limb and prosthesis, which transfers the loading during ambulation between limb and prosthesis. The correct socket design for the individual is crucial in achieving comfort and optimal use of the prosthetic limb.

This residual limb - prosthetic socket interface alters the natural demand placed upon the residual limb, requiring the loads produced during gait to be borne by the soft tissue rather than the bone. To minimise detrimental interactions between the prosthetic 
socket and residual limb, the new situation that causes severe skin and vascular problems [1], needs to be understood. The stress-strain state of this interaction has been investigated in previous studies by the use of finite element analysis (FEA) [2-8]. The development of finite element models commonly use MRI [3-4] or CT scans [5-8] to obtain the relevant bone and soft tissue geometry. FEA has been widely used to examine the aspects of lower limb prosthetics ranging from the donning procedure [6] and effects of dynamic loading [3] to multiple surgical and morphological factors [4]. However, the validity of these finite element models is reliant on the use of the correct geometry.

A number of previous FEA studies of the trans-femoral residual limb have modeled only the femur as the bony geometry without including the pelvis $[2,5-8]$. However, the pelvic region has been shown to play a substantial part in bearing the load of the patient in both common types of trans-femoral prosthetic sockets; ischial containment socket where the proximal socket design is to encase the ischial tuberosity of the pelvis and the quadrilateral socket where the proximal socket brim sits just beneath the ischial tuberosity $[9,10]$.

A better understanding of the role the pelvic bone has on the load transfer between residual limb and socket is necessary to increase overall knowledge and to inform future socket design. The objective of this study is to simulate the loading between a residual limb and prosthetic socket, both with and without the inclusion of the pelvic bone to understand the effect it has on the contact interface.

\section{Methods and Materials}

\subsection{Geometry Acquisition}

The geometry of a residual limb and internal bone were collected from a trans-femoral amputee who was 39 years of age, $173 \mathrm{~cm}$ in height with an approximate body-weight of $74 \mathrm{~kg}$. The geometries of the residual limb and bone were collected by a GE Lightspeed Plus CT scanner with a 512 x 512 pixel matrix, $0.703 \mathrm{~mm}$ pixel size, $1.25 \mathrm{~mm}$ slice increment and $0.0^{\circ}$ gantry tilt.

During the CT scan the patient was lying supine without the addition of a liner or socket. The bone and residual limb surfaces were identified and segmented using Mimics version 19.0 (Materialise, Leuven, Belgium). For the first model the bone surface was taken only as the femur (non-pelvic model), whilst in the second model the bone surface was taken as the femur and pelvic bone combined (pelvic model).

These surfaces were loaded into 3-Matic version 11.0 (Materialise, Leuven, Belgium) to convert the surfaces into solid parts. During this process the muscle, fat and skin of the soft tissue were modelled as a single entity. The internal geometry of the socket was assumed to be the same as the external surface of the residual limb, which is the approach used in previous similar studies [4, 5]. The resulting solid three-dimensional models comprised of bone, soft tissue and socket (see Fig. 1) and were meshed with 4-node tetrahedral elements. The total number of elements ranged from approximately 534,000 to 565,000 depending on model configuration. 


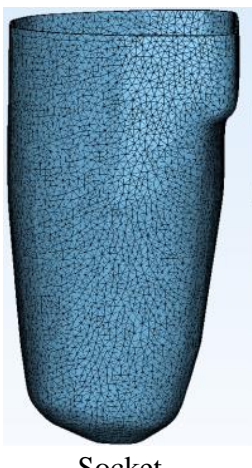

Socket

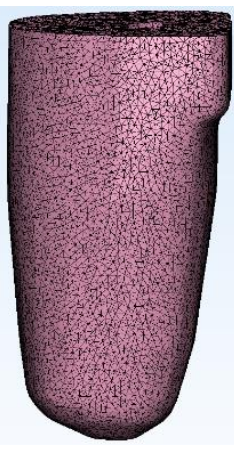

Soft tissue

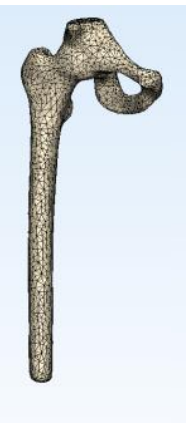

Pelvic model Non

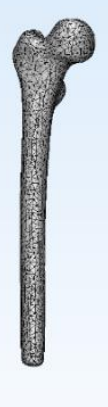

pelvic

Fig. 1. FE mesh of prosthetic socket, soft tissue, pelvic model bone and non-pelvic model bone.

\subsection{Mechanical Properties}

The bone and socket materials were considered as linear elastic, homogeneous and isotropic. In previous studies, linear elasticity was also implemented for the soft tissues with a Young's modulus of $100-400 \mathrm{kPa}$ and Poisson's ratio of $0.45-0.49$ [1-3, 8]. However, as the loading of a residual limb exhibited large deformation Portnoy et al. [4] introduced the use of a hyper-elastic model for the soft tissue to account for this. For the soft tissue hyper-elastic and linear, homogeneous and isotopic properties were defined using the $3^{\text {rd }}$ order Mooney-Rivlin strain energy function (see Table. 1). The material constants were taken from literature [3-6].

Table 1. Mechanical properties for the bone, socket and soft tissue.

\begin{tabular}{lll}
\hline & Young's Modulus (MPa) & Poisson's ratio \\
\hline Bone & 15000 & 0.3 \\
Socket & 1500 & 0.3 \\
Soft Tissue & $\mathrm{C}_{10}=0.00425, \mathrm{C}_{11}=0, \mathrm{D}_{01}=2.36$ & \\
\hline
\end{tabular}

\subsection{Loads and Boundary Conditions}

The interface between the bone and soft tissue was modeled as a tied constraint which prevents movement between the two parts. The soft tissue and socket interface was modeled as a surface to surface contact interaction using ABAQUS CAE 2017®, which prevented the slave surface (soft tissue) from penetrating the master surface (socket) during the analysis. For this interaction a friction coefficient of 0.45 was used as this was within a range of previously reported values [11].

The analysis was conducted as a two phase process. The first phase simulated the pre-stresses due to the donning procedure. This was done by applying a $50 \mathrm{~N}$ load to the distal end of the socket with the proximal region of the bone being constrained in 
movement; the proximal bone region was the femoral head for the non-pelvic model and the proximal pelvis for the pelvic model, respectively. The resulting stresses were kept in the second phase. In the second phase a walking load of $110 \%$ of the patient's bodyweight $(800 \mathrm{~N})$ was applied to the distal end of the socket with the proximal region of the bone remaining fixed.

\section{Results}

The stress distribution from the soft tissue - socket interaction was characterized in terms of contact pressure, circumferential shear stress and longitudinal shear stress (see Table 2). The pre-stress from the donning application produced a contact pressure peak of $3.48 \mathrm{kPa}$ and $5.48 \mathrm{kPa}$ for the non-pelvic and pelvic models, respectively (see Fig. 2).

Table 2. Peak values of stresses at the soft tissue-socket interface.

\begin{tabular}{|c|c|c|c|c|}
\hline Model & $\begin{array}{c}\text { Donning contact } \\
\text { pressure }(\mathrm{kPa})\end{array}$ & $\begin{array}{l}\text { Loading contact } \\
\text { pressure }(\mathrm{kPa})\end{array}$ & $\begin{array}{l}\text { Loading circumferen- } \\
\text { tial shear stress }(\mathrm{kPa})\end{array}$ & $\begin{array}{c}\text { Loading longitudinal } \\
\text { shear stress }(\mathrm{kPa})\end{array}$ \\
\hline Non-pelvic & 3.48 & 94.91 & $+20.09 /-20.89$ & $+32.79 /-25.76$ \\
\hline Pelvic & 5.48 & 364.40 & $+14.93 /-78.06$ & $+37.79 /-38.85$ \\
\hline
\end{tabular}

Fig. 2. Contact pressure at the soft tissue - socket interface due to donning. (a) Non pelvic model. (b) Pelvic model.

Figures $3 \& 4$ and Table 2 show the contact pressure and shear stress distribution and peaks generated from the second loading phase. The contact pressure peaked at 94.91 $\mathrm{kPa}$ for the non-pelvic model and was located at the distal end of the residual limb, whilst the pelvic model peaked at $364.40 \mathrm{kPa}$ and was produced at the ischial loading region. The circumferential and longitudinal shear stress for the non-pelvic model peaked at -20.89 and $32.79 \mathrm{kPa}$ respectively (see Fig. 3), with both peaks occurring at the distal end of the soft tissue. The circumferential and longitudinal shear stress for the pelvic model peaked at -78.06 and $-38.85 \mathrm{kPa}$ respectively (see Fig. 4), with both peaks occurring at the ischial loading region. 
(a)

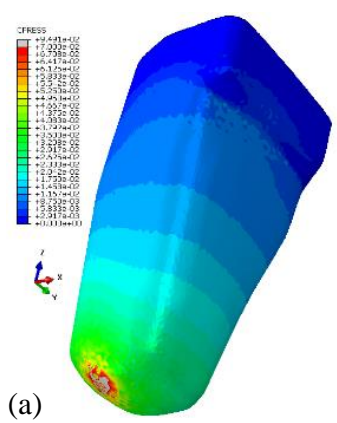

(b)

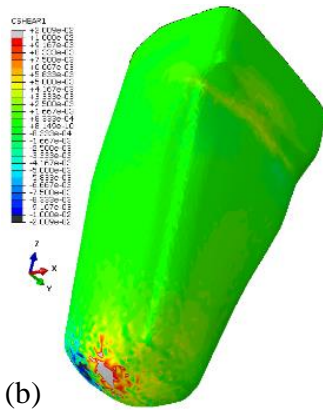

(c)

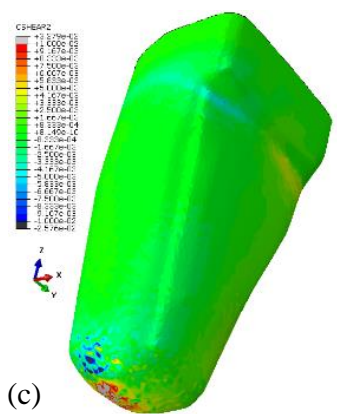

Fig. 3. (a) The contact pressure, (b) circumferential shear stress and (c) longitudinal shear stress for the non-pelvic model.
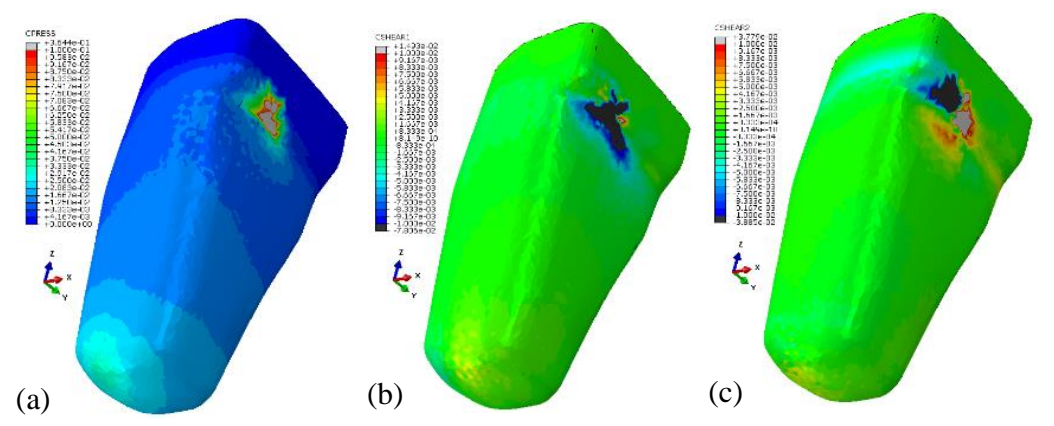

Fig. 4. (a) The contact pressure, (b) circumferential shear stress and (c) longitudinal shear stress for the pelvic model.

\section{Discussion}

In previous studies it was often assumed that the socket geometry shares the same shape as the residual limb $[4,5]$, this was also the case for this study. The difference in geometry between the socket and residual limb socket leads itself to pre-stress conditions from the donning process. However as that was absent in this study, a $50 \mathrm{~N}$ load was used to replicate this in simulation as previously implemented by Zhang et al. [5]. The resulting maximum contact pressure from this was $3.48-5.48 \mathrm{kPa}$, which is close to the maximum pressure reported by Lacroix and Patino [6] (1.54 - 5.61 kPa) who implemented an explicit finite element method to accurately simulate the donning process.

The resultant peak contact pressure of $95 \mathrm{kPa}$ for the non-pelvic model is in good agreement with the findings of Zhang and Mak [2] who reported a peak contact pressure peak of $63 \mathrm{kPa}$ at the distal end of a distal loaded trans-femoral socket.

The pelvic model in this study resulted in a peak contact pressure of $364 \mathrm{kPa}$ at the ischial support region and $36 \mathrm{kPa}$ at the distal end. Similarly, Zhang et al. [5] reported peak contact pressures of 119.30 and $80.57 \mathrm{kPa}$ at the ischial support region and distal end, respectively. The study by Zhang et al. [5] did not include the pelvic bone and 
consequently their peak contact pressures at the ischial support region were considerably lower than obtained in this study. Their resultant stresses may be attributed to the socket brim geometry and the movement constraint placed on the proximal soft tissue.

The results of an experimental study by Kahle and Highsmith [10] which measured the interface between prosthetic socket and residual limb during walking using Tekscan F-Socket System, produced a peak contact pressure of $254.67 \mathrm{kPa}$ in the ischial support region. The contact pressure and location reported by Kahle and Highsmith [10] are in agreement with those produced by the pelvic model in this study.

This study demonstrates the importance of implementing true bone geometry in the production of lower limb finite element models when examining the residual limb prosthetic socket interface.

\section{Conflict of interest}

There is no potential conflict of interest.

\section{References}

1. Zhang, M., Mak, A.F.T., Roberts, V.C.: Finite element modelling of a residual lower-limb in a prosthetic socket: a survey of the development in the first decade. Med. Eng. Phys. 20, 360-373 (1998).

2. Zhang, M., Mak, A.F.T.: A finite element analysis of the load transfer between an aboveknee residual limb and its prosthetic socket - Roles of interface friction and distal-end boundary conditions. IEEE Trans. Rehabil. Eng. 4(4), 337-346 (1996).

3. Jia, X., Zhang, M., Lee, W.: Load transfer mechanics between trans-tibial prosthetic socket and residual limb - dynamic effects. J. Biomech. 37, 1371-1377 (2004).

4. Portnoy, S., Siev-Ner, I., Yizhar, Z., Kristal, A., Shabshin, N., Gefen, A.: Surgical and morphological factors that affect internal mechanical loads in soft tissues of the transtibial residuum. Ann. Biomed. Eng. 37(12), 2583-2605 (2009).

5. Zhang, L., Zhu, M., Shen, L., Zheng, F.: Finite element analysis of the contact interface between trans-femoral stump and prosthetic socket. In: $35^{\text {th }}$ Annual International Conference of the IEEE EMBS, pp. 3-7. Osaka, Japan (2013).

6. Lacroix, D., Patino, J.F.R.: Finite element analysis of donning procedure of a prosthetic transfemoral socket. Ann. Biomed. Eng. 39(12), 2972-2983 (2011).

7. Restrepo, V., Villarraga, J., Palacio, J.P.: Stress reduction in the residual limb of a transfemoral amputee varying the coefficient of friction. J. Pros. Orthot. 26(4), 205-211 (2014).

8. Velez Zea, J.A., Goez, L.M.B., Ossa, J.A.V.: Relation between the length of the residual limb length and stress distribution over stump for transfemoral amputees. Revista EIA (Spanish) 12(23), 107-115 (2015).

9. Schuch, C.M., Pritham, C.H.: Current transfemoral sockets. Clin Orthop Relat Res 361, 4854 (1999)

10. Kahle, J., Highsmith, M.J.: Transfemoral sockets with vacuum-assisted suspension comparison of hip kinematics, socket position, contact pressure, and preference: Ischial containment versus brimless. J Rehabil Res Dev 50(9), 1241-1252 (2013)

11. Ramirez, J., Pavon, J., Toro, A.: Experimental assessment of friction coefficient between polypropylene and human skin using instrumented sclerometer. J. Eng. Tribol. 229(3), 259265 (2015). 